\title{
EVOLUTION FROM THE BCS TO THE BOSE-EINSTEIN LIMIT IN A $d$-WAVE SUPERCONDUCTOR AT $T=0$
}

\author{
L.S. Borkowski ${ }^{a, b}$ AND C.A.R. SÁ DE Melo ${ }^{b}$ \\ ${ }^{a}$ Institute of Physics, A. Mickiewicz University \\ Umultowska 85, 61-614 Poznań, Poland \\ ${ }^{b}$ School of Physics, Georgia Institute of Technology, Atlanta, Georgia 30332, USA
}

(Received January 18, 2001; in final form May 7, 2001)

\begin{abstract}
We study the evolution from BCS to Bose limit in a two-dimensional $d$-wave superconductor at zero temperature and low density of charge carriers within the mean-field theory. We examine single quasiparticle properties when particle density and attraction strength are varied. For sufficiently high interaction strength there is a critical density below which the system has a gap. The spectral and thermodynamic properties of the system do not evolve smoothly from the BCS-like to the Bose-like regime.

PACS numbers: 74.20.-z, 74.25.Gz, 67.40.Db
\end{abstract}

\section{Introduction}

The problem of the evolution from BCS to Bose superconductivity is an old one $[1,2]$ but recently it has received considerable attention in connection with high temperature superconductors [3-12]. While the effect of $d$-wave pairing on the opening of a pseudogap above $T_{c}$ was discussed in the literature, there was a lack of detailed studies of the ground state properties in the intermediate regime. It is well known that the $s$-wave system exhibits a smooth crossover between the weak and strong coupling regimes. However, pairs with non-s-wave symmetry cannot contract in real space to point bosons due to finite angular momentum of the pairs. Thus one may expect $d$-wave systems to behave in a qualitatively different way from their $s$-wave counterparts as the bosonic limit is approached. Here we discuss the single quasiparticle properties (excitation spectrum, momentum distribution, and density of states) as a function of attraction strength or particle density.

The weak coupling (BCS) limit is characterized by a positive chemical potential $\mu=\epsilon_{\mathrm{F}}$ and a large size of Cooper pairs $\left(\xi_{\text {pair }} \gg k_{\mathrm{F}}^{-1}\right)$, while the strong coupling (Bose) regime is characterized by a large and negative chemical potential $\mu=-E_{\mathrm{b}}^{(\ell)}$, where $E_{\mathrm{b}}^{(\ell)}$ is the binding energy of the two-body problem in the $\ell$-th angular momentum channel, and by a small size of pairs $\left(\xi_{\text {pair }} \ll k_{\mathrm{F}}^{-1}\right)$. 


\section{The model}

We start with the two-dimensional Hamiltonian

$$
H=\sum_{\boldsymbol{k} \sigma} \epsilon_{\boldsymbol{k}} \psi_{\boldsymbol{k} \sigma}^{\dagger} \psi_{\boldsymbol{k}_{\boldsymbol{k}} \sigma}+\sum_{\boldsymbol{k} \boldsymbol{k}^{\prime} \mathbb{\mathscr { T }}} V_{\boldsymbol{k} \boldsymbol{k}^{\prime}} b_{\boldsymbol{k} \mathscr{q}}^{\dagger} b_{\boldsymbol{k}^{\prime} \mathscr{Q}},
$$

where $b_{\mathbb{k} \mathbb{Z}}=\psi_{-\mathbb{k}+\mathbb{Z} / 2 \downarrow} \psi_{\mathbb{k}+\mathbb{Z} / 2 \uparrow}$. The interaction potential $V_{\boldsymbol{k} \mathbb{R}^{\prime}}$ is expanded in angular momentum components as $V_{\boldsymbol{k} \boldsymbol{k}^{\prime}}=\sum_{\ell=-\infty}^{+\infty} V_{\boldsymbol{k} \boldsymbol{k}^{\prime}}^{(\ell)} \exp \left(\mathrm{i} \ell \phi_{\boldsymbol{k} \boldsymbol{k}^{\prime}}\right)$, where $\phi_{\boldsymbol{k}_{\boldsymbol{k}^{\prime}}}=\arccos \left(\hat{\boldsymbol{k}} \cdot \hat{\boldsymbol{k}}^{\prime}\right)$ is the angle betwen the vectors $\boldsymbol{k}$ and $\boldsymbol{k}^{\prime}$ and $V_{k \boldsymbol{k}^{\prime}}^{(\ell)}=2 \pi \int_{0}^{\infty} \mathrm{d} r r J_{\ell}(k r) J_{\ell}\left(k^{\prime} r\right) V(r)$. The index $\ell$ labels angular momentum states in two spatial dimensions, with $\ell=0, \pm 1, \pm 2, \ldots$ corresponding to $s, p, d, \ldots$ channels respectively. A possible choice of the real space potential is $V(r)=$ $V_{1} \Theta\left(R_{1}-r\right)-V_{0} \Theta\left(r-R_{1}\right) \Theta\left(R_{0}-r\right)$, which is repulsive at short distances $r<R_{1}$, attractive at intermediate distances $R_{1}<r<R_{0}$, and vanishes for $r>R_{0}$.

Generally, it is not possible to find a separable potential in momentum space $V_{\boldsymbol{k} \boldsymbol{k}^{\prime}}=-\lambda w^{*}(\boldsymbol{k}) w\left(\boldsymbol{k}^{\prime}\right)$, nevertheless in the spirit of Ref. [2] we choose to study a separable potential that contains most of the general features described above. We consider only singlet superconductivity, where the $s$-wave and the $d$-wave channels are studied separately. We use potential of the form $V_{\boldsymbol{k} \boldsymbol{k}^{\prime}}=-\lambda_{\ell} w_{\ell}(\boldsymbol{k}) w_{\ell}\left(\boldsymbol{k}^{\prime}\right)$. The interaction term $w_{\ell}(\boldsymbol{k})$ can be written as a product of two functions, $w_{\ell}(\boldsymbol{k})=$ $h_{\ell}(k) g_{\ell}(\hat{k})$, where $h_{\ell}(k)=\left(k / k_{1}\right)^{\ell} /\left[1+\left(k / k_{0}\right)\right]^{\ell+1 / 2}$ controls the range of the interaction and $g_{\ell}(\hat{k})=\cos (\ell \phi)$ is the angular dependence of the interaction. Here $k_{0} \sim R_{0}^{-1}$ and $k_{1}$ sets the scale at low momenta. We assume that pairing at $T=0$ occurs with the same total momentum $\boldsymbol{q}=\mathbf{0}$ only. This simplification leads to the following saddle point and number equations:

$$
\begin{aligned}
& \frac{1}{\lambda_{\ell}}=\sum_{\boldsymbol{k}} \frac{\left|w_{\ell}(\boldsymbol{k})\right|^{2}}{2 E_{\ell}(\boldsymbol{k})}, \\
& n=2 \sum_{\boldsymbol{k}} n_{\ell}(\boldsymbol{k}),
\end{aligned}
$$

where $n_{\ell}(\boldsymbol{k})=\left[1-\left(\epsilon_{\boldsymbol{k}}-\mu\right) / E_{\ell}(\boldsymbol{k})\right] / 2$ is the momentum distribution, $E_{\ell}(\boldsymbol{k})=$ $\left[\left(\epsilon_{\boldsymbol{k}}-\mu\right)^{2}+\left|\Delta_{\ell}(k)\right|^{2}\right]^{1 / 2}$ is the single particle excitation energy, and $\Delta_{\ell}(k)=$ $\Delta_{0 \ell} w_{\ell}(k)$ is the order parameter. For a given interaction range $R_{0} \sim k_{0}^{-1}$, the transition from the BCS limit (largely overlapping pairs) to the Bose limit of (weakly overlapping pairs) may occur either by changing the attraction strength $\lambda_{\ell}$ or the density $n$. In either case, this evolution can be safely analyzed with the approximations used here provided that the system is dilute enough, i.e., $n \ll k_{0}^{2}$. This means that below a maximum density $n_{\max } \sim k_{\mathrm{F} \text { max }}^{2}$, the interaction range $R_{0}$ is much smaller than the interparticle spacing $k_{\mathrm{F} \max }^{-1}, R_{0} \ll k_{\mathrm{F} \max }^{-1}$, or equivalently $k_{0} / k_{\mathrm{F} \text { max }} \gg 1$. Thus we choose to scale all energies with respect to the maximum Fermi energy $\epsilon_{\mathrm{F} \max }$, which fixes the maximum density $n=n_{\max }=2 \rho \epsilon_{\mathrm{F} \max }$, and all momenta with respect to $k_{\mathrm{F} \max }=\sqrt{2 m \epsilon_{\mathrm{F} \max }}$. The coupling constant is scaled with respect to the two-dimensional density of states $\rho$. From now on we use this scaling. 


\section{Results}

Numerical solutions for $\Delta_{0 \ell}$ and $\mu$, when $k_{1}=k_{0}=10$ are shown in Fig. 1 for fixed density $n=1$, and changing $\lambda_{\ell}$. Similar plots can also be made for fixed interaction and varying density $n$. In the weak coupling limit the amplitude of the order parameter $(\phi=0)$ is given by

$$
\Delta_{\ell}\left(k_{\mu}\right) \sim \exp \left\{2\left[\lambda_{0 \ell}^{-1}\left(k_{\mu}\right)-\lambda_{\ell}^{-1}\right] / h_{\ell}^{2}\left(k_{\mu}\right)\right\} .
$$

With our choice of $h_{\ell}(k), \lambda_{0 d}\left(k_{\mu}\right) \simeq 8+\mu / 24 \epsilon_{1}+\mathcal{O}\left[\left(\mu / \epsilon_{1}\right)^{2}\right]$, valid for $\mu / \epsilon_{1} \ll 1$, where $\epsilon_{1}=k_{1}^{2}$. The ratios between $\Delta_{\ell}\left(k_{\mu}\right)$ and the critical temperature $T_{c \ell}$ satisfy the usual relations $\Delta_{s}\left(k_{\mu}\right) / T_{\mathrm{cs}}=1.76$, and $\Delta_{d}\left(k_{\mu}\right) / T_{\mathrm{cd}}=2.14$. The parameters $\Delta_{0 d}$ and $\mu$ have continuous first derivatives and discontinuous second derivatives as a function of $\lambda_{d}$. This behavior always occurs when $\mu=0$ in both $\Delta_{0 d}$ and $\mu$, for varying interaction $\lambda_{d}$ (see Fig. 1) or varying density $n$.
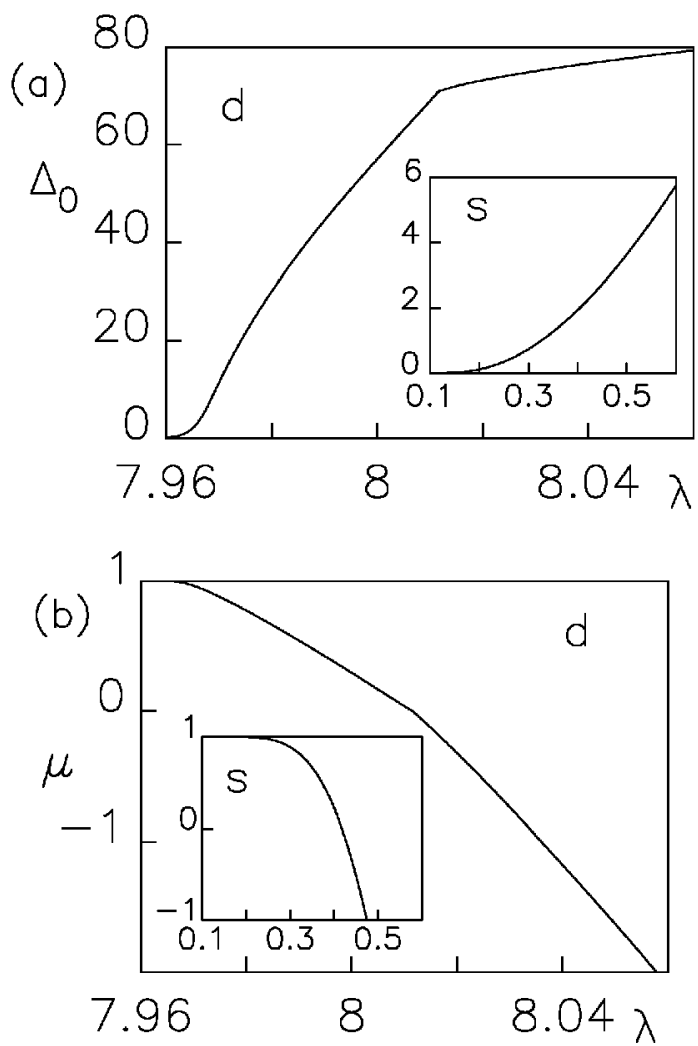

Fig. 1. (a) The order parameter $\Delta_{0}$ and (b) the chemical potential $\mu$ as a function of coupling at fixed density $n=1$ and $k_{1}=k_{0}=10$ for both $s$-and $d$-wave channels. In the $d$-wave case $\Delta_{0}(\lambda)$ and $\mu(\lambda)$ have continuous first derivatives and discontinuous second derivatives at $\mu=0$. 
We first look at the single quasiparticle excitation spectrum $E_{d}(\boldsymbol{k})$. For $\mu>0$, including the BCS limit, the excitation spectrum is gapless at $k_{\mu}$ along the special directions $\phi= \pm \pi / 4, \pm 3 \pi / 4$, near which the excitation spectrum disperses linearly with momentum. The energy gap at $k=k_{\mu}$ and $\phi=0, E_{\mathrm{g}}\left(k_{\mu}\right)=\left|\Delta_{d}\left(k_{\mu}\right)\right|$ is a nonmonotonic function of $k_{\mu}$ for fixed density, and thus a nonmonotonic function of $\lambda_{d}$. The maximum $E_{\mathrm{g}}\left(k_{\mu}\right)$ is reached at intermediate values of $\mu>0$. At $\mu=0$, the minimum gap is $E_{\mathrm{g}}(0)=\left|\Delta_{d}(0)\right|=0$, and occurs at the single point $k=0$. In this case the excitation spectrum is $E_{d}(\boldsymbol{k})=\left(\epsilon_{\boldsymbol{k}}^{2}+\left|\Delta_{d}(\boldsymbol{k})\right|^{2}\right)^{1 / 2}$, which behaves quadratically for small momenta at any given angle $\phi$, since $\Delta_{d}(k) \sim k^{2} \cos (2 \phi)$ and $\epsilon_{\boldsymbol{k}}=k^{2} / 2 m$. The shrinking of the energy gap to zero at $k=\mathbf{0}$ is a consequence of the diminishing pairing interaction $h_{d}\left(k_{\mu}\right)$ for $\mu \rightarrow 0$. As soon as $\mu<0$, including the Bose limit, a full gap in the excitation spectrum appears, but the minimal gap remains at $k=0, E_{\mathrm{g}}(0)=|\mu|$, since $\Delta_{d}(0)=0$, see Fig. $2[3]$.

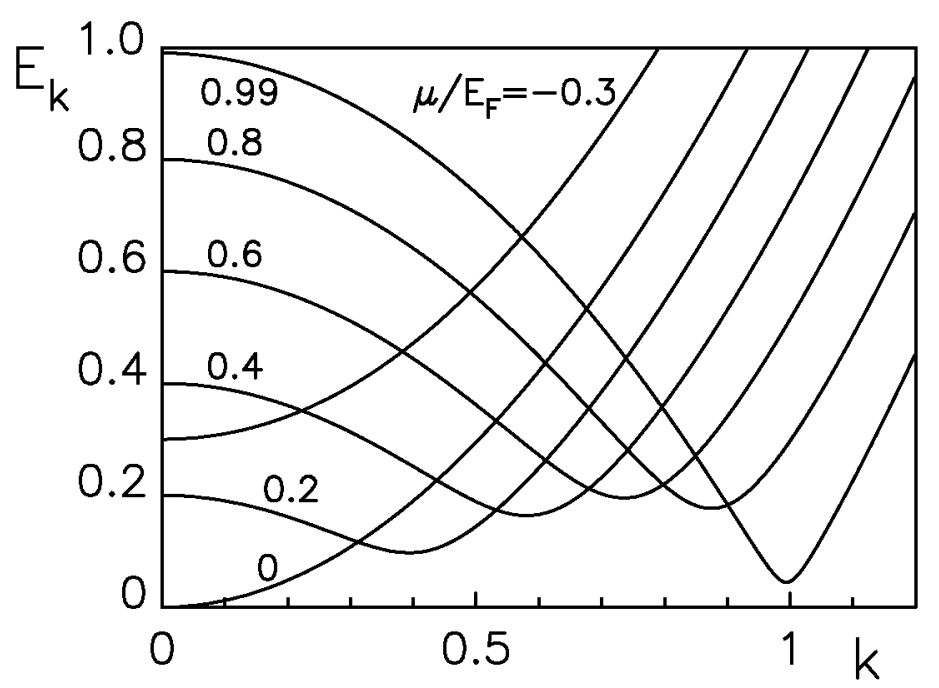

Fig. 2. Energy gap as a function of momentum along the direction $\phi=0 ; k_{1}=k_{0}=10$.

Figure 3 shows the lines where $\mu=0$ on the graph of $n$ vs. $\lambda_{\ell}$. The low density limit of the $s$-wave system is always Bose-like, i.e., a two-body bound state appears at arbitrarily small $\lambda_{s}$. The $d$-wave system is qualitatively different: it is BCS-like for $\lambda_{d}<\lambda_{c d}$ and Bose-like for $\lambda_{d}>\lambda_{c d}$, where the critical coupling $\lambda_{\mathrm{c}}$ separating the two regimes is finite, i.e., the appearance of a two-body bound state in the $d$-wave case requires finite $\lambda_{d}$.

Let us briefly discuss the behavior of the momentum distribution at low $k$ for three different regimes: $\mu>0, \mu=0$, and $\mu<0$. For positive $\mu$ the momentum distribution is $n_{s}\left(k_{\mu}+\delta k\right) \simeq\left[1-2 k_{\mu} \delta k / \Delta_{s}\left(k_{\mu}\right)\right] / 2$ near $k_{\mu}$. At low $k$, however, $n_{s}(k) \simeq\left[1+\gamma_{p}\left(1+\alpha k / 2 k_{0}\right)\right] / 2$, where $\gamma_{p}=\mu / \sqrt{\mu^{2}+\Delta_{0 s}^{2}}$, and $\alpha=\Delta_{s}^{2} /\left(\mu^{2}+\Delta_{0 s}^{2}\right)$. For $\mu=0$ and small $k, n_{s}(k) \simeq\left(1-k^{2} / \Delta_{0 s}\right) / 2$. For negative $\mu, n_{s}(k)=\left[1-\gamma_{n}\left(1+\alpha k / 2 k_{0}\right)\right] / 2$ for small $k$, with $\gamma_{n}=|\mu| / \sqrt{\mu^{2}+\Delta_{0 s}^{2}}$. Obviously, $n_{s}(k)$ is a continuous function of $\mu$ for all $k$. 


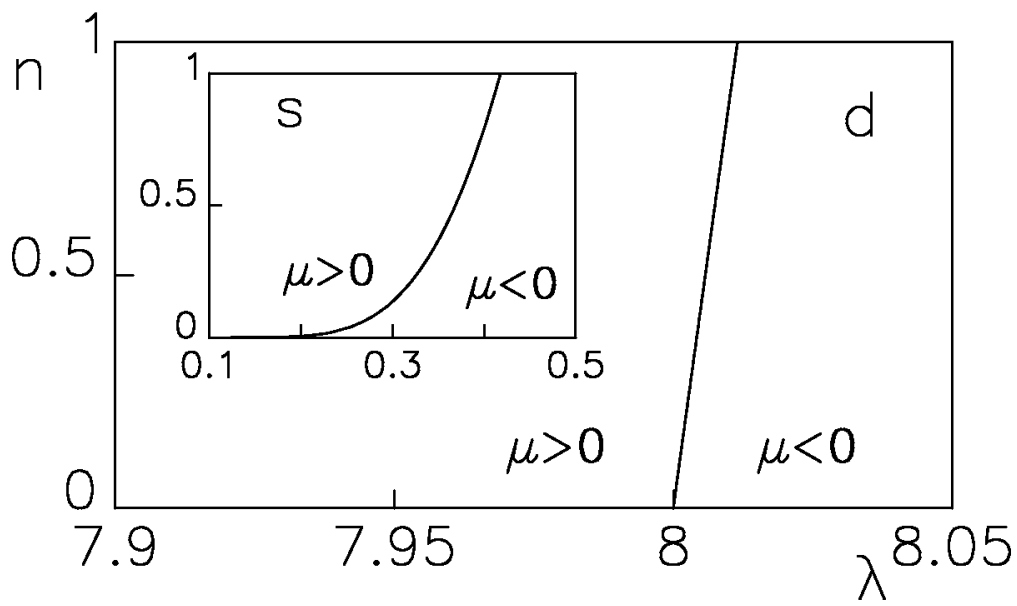

Fig. 3. The line $\mu=0$ for both $s$ - and $d$-wave order parameters for $k_{1}=k_{0}=10$.

The momentum distribution in the $d$-wave case has the form $n_{d}(k)=\left[1-\operatorname{sgn}\left(k^{2}-\mu\right)\right]$ along the direction of the nodes $(\phi= \pm \pi / 4, \pm 3 \pi / 4)$. Near $k_{\mu}$ we have $n_{d}\left(k_{\mu}+\delta k\right) \simeq\left[1-2 k_{\mu} \delta k / \Delta_{d}\left(k_{\mu}\right)\right] / 2$, for $k$ close to $k_{\mu}$, and $n_{d}(k) \simeq 1-\left(\Delta_{0 d}^{2} / \mu^{2}\right)\left(k^{4} / 4 k_{1}^{4}\right)$ for small $k$. When $\mu$ vanishes, at $k=0$ is $n_{d}(0) \simeq(1-\kappa) / 2$, where $\kappa=\left(1+\Delta_{0 d}^{2} / k_{1}^{4}\right)^{-1 / 2}$. Finally, when $\mu$ becomes negative, $n_{d}(k) \simeq\left(\Delta_{0 d}^{2} / \mu^{2}\right)\left(k^{4} / 4 k_{1}^{4}\right)$ for small $k$. The discontinuity of $n_{d}(k)$ at $\mu=0$ and low $k$, see Fig. 4 , coincides with the collapse of the four Dirac points to a single point at $k_{\mu}=0$, and with the appearance of a full gap as soon as $\mu<0$. Similar behavior of $n_{d}(k)$ was also found recently in Ref. [13, 14] for a lattice model with attractive interaction of nearest-neighbor particles.

The qualitative changes in $E_{\ell}(\boldsymbol{k})$ and $n_{\ell}(\boldsymbol{k})$, as a function of $\mu$, affect substantially the quasiparticle density of states $N_{\ell}(\omega)=N_{\ell}^{(+)}(\omega)+N_{\ell}^{(-)}(\omega)$, where

$$
N_{\ell}^{(+)}(\omega)=(2 \pi)^{-1} \int \mathrm{d}^{2} \boldsymbol{k}\left[1-n_{\ell}(\boldsymbol{k})\right] \delta\left[\omega-E_{\ell}(\boldsymbol{k})\right]
$$

corresponds to adding a quasiparticle, and

$$
N_{\ell}^{(-)}(\omega)=(2 \pi)^{-1} \int \mathrm{d}^{2} \boldsymbol{k} n_{\ell}(\boldsymbol{k}) \delta\left[\omega+E_{\ell}(\boldsymbol{k})\right]
$$

corresponds to removing a quasiparticle. At low frequencies $N_{d}(\omega)$ changes discontinuously from linear in $\omega$ for $\mu>0$, where $E_{d}(\boldsymbol{k})$ is linear in momentum close to the nodes, to a constant at $\mu=0$ (where $E_{d}(k) \propto k^{2}$ at low $k$ ), to zero for $\mu<0$ (where $E_{d}(\boldsymbol{k}) \simeq|\mu|+\mathcal{O}\left(k^{2}\right)$ for small $k$ ), as can be seen in Fig. 5 . In the calculation of the density of states we have neglected the effects of quasiparticle lifetimes*. The lack of particle-hole symmetry seen in Fig. 5 is a general property

*These lifetime effects come from quantum fluctuations which introduce self-energy corrections to the single quasiparticle propagator. The self-energy corrections originate from quasiparticle-quasiparticle and quasiparticle-quasihole interactions and are quite important in the high density limit $\left(n \sim k_{0}^{2}\right.$ ), however at low densities ( $n \ll k_{0}^{2}$, the only situation discussed in this manuscript) lifetime broadenings scale with $n / k_{0}^{2}$ and do not contribute substantially to the line shapes at low frequencies and low momenta. 


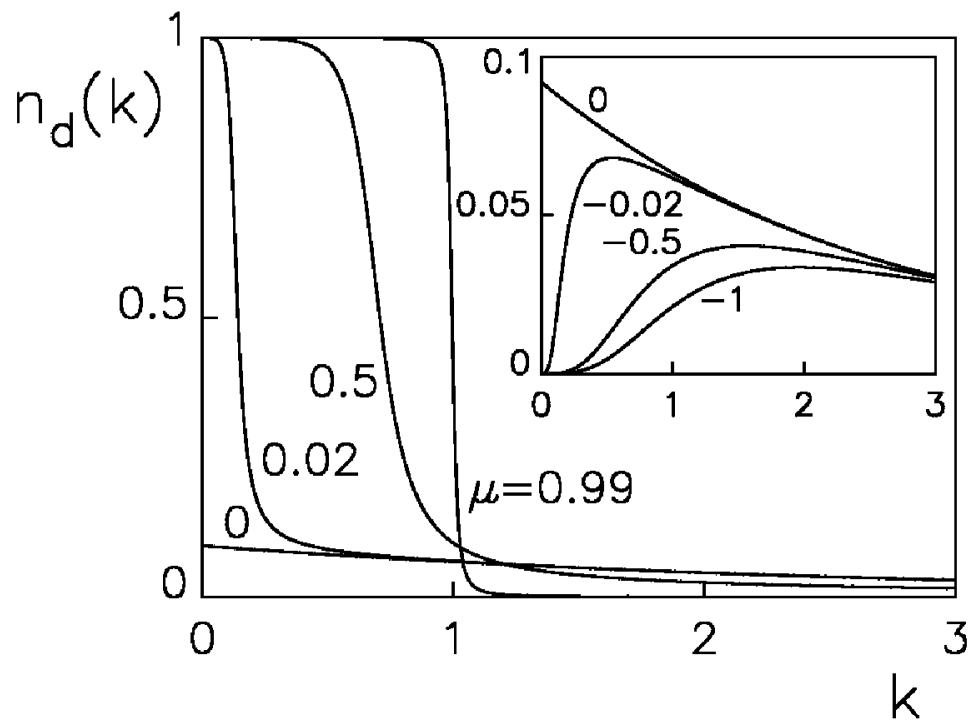

Fig. 4. The momentum distribution of quasiparticles for $\phi=0, n=1, k_{1}=k_{0}=10$, and several values of $\mu$ for a $d$-wave order parameter. The inset shows results for $\mu \leq 0$.

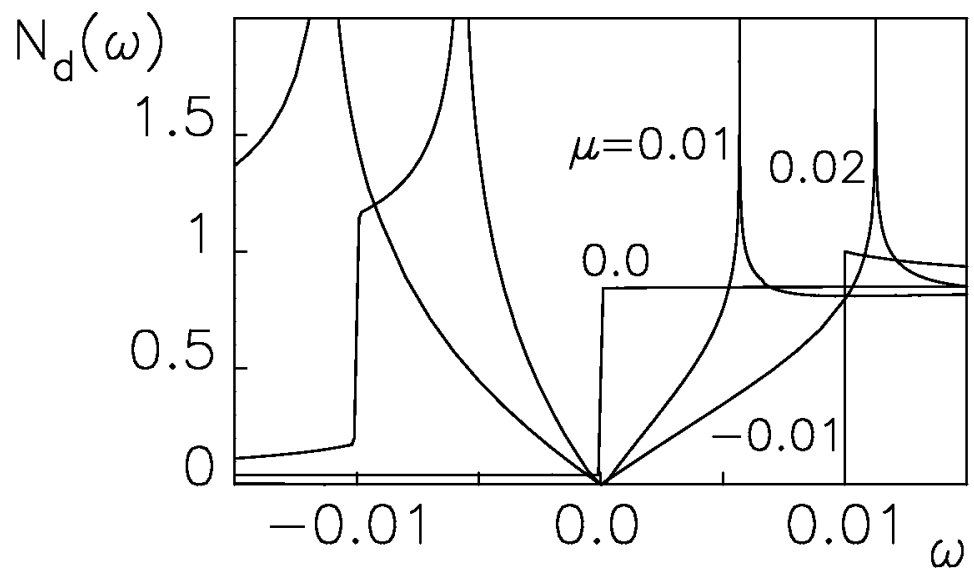

Fig. 5. Density of states for a $d$-wave order parameter near $\mu=0$, for $n=1, k_{1}=$ $k_{0}=10$, and varying $\lambda_{d}$.

of superconducting systems with small chemical potential (see Ref. [15] for results in the normal state of a boson-fermion model in the regime of positive $\mu$ ).

The contributions from quasiparticles to specific heat $C$ and spin susceptibility $\chi$ change from $C \propto T^{2}$, and $\chi \propto T$ for $\mu>0$, to $C \propto T$, and $\chi \propto$ const for $\mu=0$, and to $C \propto T^{-1} \exp (-|\mu| / T)$, and $\chi \propto \exp (-|\mu| / T)$ for $\mu<0$. The slopes of $C$ and $\chi$ with respect to temperature are discontinuous at $T=0$ when $\mu=0$. 


\section{Conclusions}

In summary, we studied the evolution from BCS to Bose limit for varying interaction strength in a $d$-wave superconductor. The ground state properties of this system change significantly when the chemical potential $\mu$ changes sign. The entire momentum distribution $n_{d}(\boldsymbol{k})$ is redistributed, with largest changes occurring at low $k$. This reorganization in momentum space is related to the transition from an extended to a local character of the pair wave function. The symmetry of the wave function is preserved but its topology is altered ${ }^{\dagger}$. The character of spectroscopic and thermodynamic properties changes from a power-law to an exponential behavior, as $\mu$ becomes negative.

For constant pairing strength $\lambda$ and varying particle density, quantities such as pair size, correlation length and compressibility diverge at $\mu=0$ in the saddle-point approximation. This might indicate the existence of a quantum phase transition. We will publish these and other results separately [16]. In order to answer the question whether these discontinuities indicate the quantum phase transition or are simply an artifact of the mean-field theory one needs to include the finite lifetimes of the quasiparticles.

\section{Acknowledgments}

We would like to thank the Georgia Institute of Technology for financial support. Some of the numerical calculations were performed at the Poznań Supercomputer and Networking Center (PCSS). An earlier version of this paper is available in the xxx.lanl.gov archive as preprint cond-mat/9810370.

\section{References}

[1] A.J. Leggett, in: Modern Trends in the Theory of Condensed Matter, Eds. A. Pekalski, J. Przystawa, Springer-Verlag, Berlin 1980; J. Phys. (Paris) Colloq. 41, C7-19 (1980).

[2] P. Nozières, S. Schmitt-Rink, J. Low-Temp. Phys. 59, 195 (1985).

[3] M. Randeria, J.-M. Duan, L.-Y. Shieh, Phys. Rev. B 41, 327 (1990).

[4] S. Schmitt-Rink, C.M. Varma, A.E. Ruckenstein, Phys. Rev. Lett. 63, 445 (1989).

[5] R. Micnas, J. Ranninger, S. Robaszkiewicz, Rev. Mod. Phys. 62, 113 (1990).

[6] M. Randeria, in: Bose-Einstein Condensation, Eds. A. Griffin, D. Snoke, S. Stringari, Cambridge Univ. Press, Cambridge 1995, p. 355.

[7] M. Drechsler, W. Zwerger, Ann. Phys. 1, 15 (1992); S. Stintzing, W. Zwerger, Phys. Rev. B 56, 9004 (1997).

[8] F. Pistolesi, G.C. Strinati, Phys. Rev. B 53, 15168 (1996).

${ }^{\dagger}$ The existence of a topological quantum phase transition in ${ }^{3} \mathrm{He}$, a $p$-wa ve superfluid, at $\mu=0$ in the $n$ vs. $\lambda$ plane was discussed by G.E. Volovik, Exotic Properties of Superfluid ${ }^{3} H e$, World Scientific, Singapore 1992. In the case of ${ }^{3} \mathrm{He}$ the transition is associated with the change of symmetry of the ground state. In a $d$-wave superconductor, the transition would occur without the change of symmetry. 
[9] B. Janko, J. Maly, K. Levin, Phys. Rev. B 56, R11407 (1997).

[10] S.K. Adhikari, A. Ghosh, Phys. Rev. B 55, 1110 (1997).

[11] C.A.R. Sá de Melo, M. Randeria, J.R. Engelbrecht, Phys. Rev. Lett. 71, 3202 (1993); J.R. Engelbrecht, M. Randeria, C.A.R. Sá de Melo, Phys. Rev. B 55, 15153 (1997).

[12] J.R. Engelbrecht, A. Nazarenko, M. Randeria, E. Dagotto, Phys. Rev. B 57, 13406 (1998).

[13] B.C. den Hertog, Phys. Rev. B 60, 559 (1999).

[14] N. Adrenacci, A. Perali, P. Pieri, G.C. Strinati, Phys. Rev. B 60, 12410 (1999).

[15] J. Ranninger, J.M. Robin, M. Eschrig, Phys. Rev. Lett. 74, 4027 (1995).

[16] L.S. Borkowski, C.A.R. Sá de Melo, to be published. 